PROCEEDINGS OF THE

AMERICAN MATHEMATICAL SOCIETY

Volume 124, Number 9, September 1996

\title{
POLYNOMIALLY BOUNDED OPERATORS AND EXT GROUPS
}

\author{
SARAH H. FERGUSON
}

(Communicated by Palle E. T. Jorgensen)

\begin{abstract}
In this paper, we consider the Ext functor in the category of Hilbert modules over the disk algebra. We characterize the group $\operatorname{Ext}_{A(D)}(K, H)$ as a quotient of operators and explicitly calculate $\operatorname{Ext}_{A(D)}\left(K, H^{2}\right)$, where $K$ is a weighted Hardy space. We then use our results to give a simple proof of a result due to Bourgain.
\end{abstract}

\section{INTRODUCTION}

In 1974, Foias and Williams studied the class of $2 \times 2$ operator matrices of the form below, [5]. Although their paper was never published, the main results appear in [3]. Foias and Williams conjectured that an operator of the form

$$
R_{f}=\left(\begin{array}{cc}
S^{*} & \Gamma_{f} \\
0 & S
\end{array}\right)
$$

where $S$ is the forward shift on $\ell^{2}$ and $\Gamma_{f}$ is the Hankel matrix with symbol $f$, is a counterexample to Halmos' famous problem: Is every polynomially bounded operator similar to a contraction?

What Foias and Williams proved was that $R_{f}$ is similar to a contraction if and only if there is a bounded solution to the commutator equation $\Gamma_{f}=S^{*} X-X S$. This means that $R_{f}$ is similar to a contraction if and only if $R_{f}$ is similar to $S^{*} \oplus S$ via a similarity of the form $\left(\begin{array}{cc}I & X \\ 0 & I\end{array}\right)$. By solving the commutator equation above, one sees that all solutions have the form $X=\Gamma_{g}-\Gamma_{f} D S^{*}$, where $g \in H^{2}$ and $D$ is the diagonal matrix $\operatorname{diag}(i+1)_{i>0}$. Paulsen observed that if $X$ is a solution to the commutator equation, then $-X^{t}$ is a solution as well. Hence

$$
Y=\frac{X-X^{t}}{2}=\left(\frac{(i-j)}{2} \hat{f}(i+j-1)\right)_{i, j \geq 0}
$$

is a bounded solution. Here $\hat{f}(n)$ is the $n^{\text {th }}$ Fourier coefficient of $f$ and $\hat{f}(-1)=0$. It follows that $R_{f}$ is similar to a contraction if and only if the matrix $Y$ is bounded on $\ell^{2}$.

Several other people have studied this operator including Peller who, in [6], proved that $R_{f}$ is power bounded if and only if $f^{\prime}$ is in the Bloch class. He also

Received by the editors March 13, 1995.

1991 Mathematics Subject Classification. Primary 47B38; Secondary 18 G15.

Key words and phrases. Polynomially bounded operator, Ext groups, reproducing kernel Hilbert space, weighted Hardy space, BMOA. 
showed that if $f^{\prime}$ is BMOA, then $R_{f}$ is polynomially bounded. Bourgain showed in [1] that if $f^{\prime}$ is BMOA, then $R_{f}$ is similar to a contraction.

An operator $T$ is polynomially bounded on $H$ if and only if the map $p \mapsto p(T)$ defined on polynomials extends to a representation of the disk algebra, $\mathbf{A}(\mathbf{D})$, on $H$. In terms of Hilbert modules, this means that the map $(p, h) \mapsto p(T) h$ extends to a Hilbert A(D)-module action on $H$. The first systematic study of Hilbert modules was done by Douglas and Paulsen in [4]. Carlson and Clark were the first to study the Ext functor in this category [2],[3].

In this paper we give a concrete characterization of $\operatorname{Ext}_{\mathcal{A}}(K, H)$ as a quotient of operators and use this together with a result from [2] to calculate the groups $\operatorname{Ext}_{A(D)}\left(K, H^{2}\right)$ for a large class of Hilbert modules $K$. We then show how these results can be used to give an alternative proof of Bourgain's result mentioned above.

\section{Homological PRELIMINARIES}

A Hilbert module $H$ over a function algebra $\mathcal{A}$ is a Hilbert space together with a bounded, unital homomorphism $\pi: \mathcal{A} \rightarrow \mathcal{L}(H)$. Such a map is called a representation of the algebra $\mathcal{A}$ on $H$. Given a representation $\pi$, one defines the module action on $H$ by $a \cdot h=\pi(a) h$. It is easy to see that every Hilbert module action arises this way. In fact, if $\rho: \mathcal{A} \times H \rightarrow H$ defines a bounded module action on $H$ then $\pi(a) h \equiv \rho(a, h)$ defines a representation of $\mathcal{A}$ on $H$.

Given two Hilbert $\mathcal{A}$-modules, $\left(H, \pi_{1}\right)$ and $\left(K, \pi_{2}\right)$, an operator $T \in \mathcal{L}(H, K)$ is called a Hilbert module map if $T \pi_{1}(a)=\pi_{2}(a) T$ for all $a \in \mathcal{A}$. $\operatorname{Ext}_{\mathcal{A}}(K, H)$ is defined to be the collection of equivalence classes of short exact sequences of the form

$$
0 \rightarrow H \stackrel{\alpha}{\rightarrow} J \stackrel{\beta}{\rightarrow} K \rightarrow 0
$$

Here $J$ is a Hilbert $\mathcal{A}$-module, $\alpha$ and $\beta$ are Hilbert module maps and exactness means that $\alpha$ is $1-1, \beta$ is onto and the range of $\alpha$ is equal to the kernel of $\beta$. We call two such sequences equivalent if there exists a Hilbert module map between the two middle modules such that the following diagram commutes:

$$
\begin{array}{ccccccccc}
0 & \rightarrow & H & \stackrel{\alpha}{\rightarrow} & J & \stackrel{\beta}{\rightarrow} & K & \rightarrow & 0 \\
& \| & & \downarrow & & \| & & \\
0 & \rightarrow & H & \stackrel{\alpha^{\prime}}{\rightarrow} & J^{\prime} & \stackrel{\beta^{\prime}}{\rightarrow} & K & \rightarrow & 0
\end{array}
$$

In this category, every short exact sequence is equivalent to one of the form

$$
0 \rightarrow H \stackrel{\iota}{\rightarrow} H \oplus K \stackrel{P}{\rightarrow} K \rightarrow 0 .
$$

Here the middle module is the Hilbert space direct sum (with an appropriately defined module action), $\iota$ is the isometric inclusion and $P$ is the orthogonal projection onto $K$. To see this fact, note from $(1)$ we have $\alpha(H)=\operatorname{kernel}(\beta)$ so that $\alpha(H)$ is closed in $J$. As a Hilbert space then, $J=\alpha(H) \oplus \alpha(H)^{\perp}$. Since the restriction of $\beta$ to $\alpha(H)^{\perp}$ maps 1-1 and onto $K, \beta$ has a right inverse $T$. Now define $S \in \mathcal{L}(H \oplus K, J)$ by $S(h+k)=\alpha(h)+T(k)$. If $\pi$ is the representation of $\mathcal{A}$ on $J$, then $\tilde{\pi}(a) \equiv S^{-1} \pi(a) S$ defines a representation of $\mathcal{A}$ on $H \oplus K$ such that the 
following diagram commutes:

$$
\begin{aligned}
& 0 \rightarrow \underset{\|}{H} \stackrel{\iota}{\rightarrow} \underset{\downarrow S}{H \oplus K} \stackrel{\rightarrow}{\rightarrow} \underset{\|}{K} \quad \rightarrow \quad 0 \\
& 0 \rightarrow H \stackrel{\alpha}{\longrightarrow} \quad J \quad \stackrel{\beta}{\longrightarrow} K \rightarrow 0
\end{aligned}
$$

If the sequence $0 \rightarrow H \stackrel{\iota}{\rightarrow} H \oplus K \stackrel{P}{\rightarrow} K \rightarrow 0$ is exact and $\pi$ is the representation on $H \oplus K$, then for each $a \in \mathcal{A}$ we get the decomposition

$$
\pi(a)=\left(\begin{array}{cc}
\pi_{1}(a) & \delta(a) \\
0 & \pi_{2}(a)
\end{array}\right)
$$

where $\pi_{1}, \pi_{2}$ are the representations on $H$ and $K$, respectively, and $\delta: \mathcal{A} \rightarrow \mathcal{L}(K, H)$ is a derivation. A derivation $\delta$ is called inner if there is an operator $X$ in $\mathcal{L}(K, H)$ such that $\delta(a)=\pi_{1}(a) X-X \pi_{2}(a), a \in \mathcal{A}$. It is easy to see that the derivation $\delta$ is inner if and only if the sequence above is equivalent to the trivial sequence (i.e., the sequence where the module action on the direct sum is $\left.\pi_{1} \oplus \pi_{2}\right)$. By identifying the representation with the derivation one gets the usual Hochschild characterization of $\operatorname{Ext}_{\mathcal{A}}(H, K)$ as derivations modulo inner ones.

\section{Ext over the DISK ALGEBRA}

Recall that an operator $T$ on a Hilbert space $H$ is polynomially bounded if and only if $p \mapsto p(T)$ extends to a representation of the disk algebra, $\mathbf{A}(\mathbf{D})$, on $H$. On the other hand, given a representation $\pi: \mathbf{A}(\mathbf{D}) \rightarrow \mathcal{L}(H)$, the operator $T=\pi(z)$ is polynomially bounded, where $z$ is the function $z \mapsto z$. Note that $\pi(p)=p(T)$ for all polynomials $p$. Because of this correspondence we will write $(H, T)$ for the Hilbert module $H$ with multiplication by $z$ determined by the operator $T$.

Let $\left(H, T_{0}\right)$ and $\left(K, T_{1}\right)$ be two Hilbert $\mathbf{A}(\mathbf{D})$-modules. A derivation $\delta: \mathbf{A}(\mathbf{D}) \rightarrow$ $\mathcal{L}(K, H)$ is uniquely determined by the operator $X=\delta(z)$ which, in turn, uniquely determines multiplication by $z$ on $H \oplus K$. It is not hard to see that $\delta$ is inner exactly when there is a $Y \in \mathcal{L}(K, H)$ such that $X=T_{0} Y-Y T_{1}$.

Let $\operatorname{PB}(K, H)$ denote the set of all $X \in \mathcal{L}(K, H)$ such that the $2 \times 2$ operator matrix

$$
\left(\begin{array}{cc}
T_{0} & X \\
0 & T_{1}
\end{array}\right)
$$

is bounded on $\mathcal{L}(H \oplus K)$, and let $\triangle(K, H)$ be the set of all commutators $T_{0} \mathrm{Y}^{-\mathrm{YT}_{1}}$ as $Y$ ranges over $\mathcal{L}(K, H)$. It follows that $\operatorname{Ext}_{A(D)}\left(K, H^{2}\right)$ is isomorphic to the quotient $\mathrm{PB}(K, H) / \triangle(K, H)$.

$$
\text { 4. } \operatorname{Ext}_{A(D)}\left(K, H^{2}\right)
$$

The Hardy space, $H^{2}$, is the Hilbert space of analytic functions on the disk satisfying

$$
\|f\|^{2} \equiv \sup _{0<r<1} \frac{1}{2 \pi} \int_{0}^{2 \pi}\left|f\left(r e^{i \theta}\right)\right|^{2} d \theta<\infty .
$$

We will frequently identify $f \in H^{2}$ with its boundary values on the circle $\mathbf{T}$. $P_{+}$will denote the orthogonal projection of $L^{2}(\mathbf{T})$ onto $H^{2}$ and $S$ will denote the forward shift on $H^{2}$. That is, $S f(z)=z f(z), f \in H^{2}$. The operator $S$ is contractive on $H^{2}$ so, by von Neumann's inequality, $\left(H^{2}, S\right)$ is a Hilbert $\mathbf{A}(\mathbf{D})$-module and the action is just pointwise multiplication. 
Recall, for $k \in K$ the rank one operator $1 \otimes k \in \mathcal{L}\left(K, H^{2}\right)$ is defined by $(1 \otimes k) f=$ $\langle f, k\rangle_{K} e_{0}$, where $e_{0}$ is the constant function 1.

The proof of the following theorem appears in [2] and allows one to calculate the

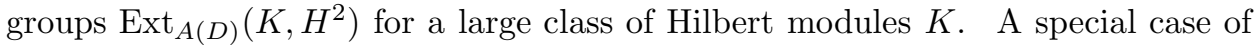
the theorem appeared in [5].

Theorem 1. Let $T$ be a polynomially bounded operator on $K$. For $X$ in $\mathcal{L}\left(K, H^{2}\right)$, let

$$
R(X)=\left(\begin{array}{cc}
S & X \\
0 & T
\end{array}\right)
$$

The following are equivalent:

1) $R(X)$ is power bounded on $H^{2} \oplus K$.

2) $R(X)$ is polynomially bounded on $H^{2} \oplus K$.

3) $\exists k \in K$ and $Y \in \mathcal{L}\left(K, H^{2}\right)$ such that

i) $X=1 \otimes k+S Y-Y T$, and

ii) For all $f \in K, \sum_{n=0}^{\infty}\left|\left\langle T^{n} f, k\right\rangle\right|^{2}<\infty$.

Remarks. (a) The first condition in 3$)$ says that $R(X)$ is similar to $R(1 \otimes k)$ via the similarity $\left(\begin{array}{cc}I & Y \\ 0 & I\end{array}\right)$. Condition ii) is precisely the condition that $R(1 \otimes k)$ is power bounded.

(b) This theorem tells us that the equivalence class of the operator $X$ in $\operatorname{Ext}_{A(D)}\left(K, H^{2}\right)$ is determined by a rank one operator of the form $1 \otimes k$ for some $k \in K$.

(c) The second condition in 3) is equivalent to the following: There exists $W \in$ $\mathcal{L}\left(H^{2}, K\right)$ such that $W S=T^{*} W$ and $W(1)=k$. To see this note that if $W$ intertwines $S$ and $T^{*}$ and sends 1 to $k$, then $W\left(e_{n}\right)=T^{* n} k$. So for $f \in K$, $\left\|W^{*} f\right\|^{2}=\sum_{n=0}^{\infty}\left|\left\langle f, W e_{n}\right\rangle_{K}\right|^{2}=\sum_{n=0}^{\infty}\left|\left\langle T^{n} f, k\right\rangle_{K}\right|^{2}$.

(d) By remark (c), if we replace $T$ by $T^{*}$ in the theorem, then $R(1 \otimes k)$ is polynomially bounded if and only if $p \mapsto p(T) k$ extends to a bounded operator from $H^{2}$ into $K$. So if $K$ is a functional Hilbert space such that $T f(z) \equiv z f(z)$ is polynomially bounded, then the theorem gives us an alternative criterion for a function $k \in K$ to be an analytic multiplier of $H^{2}$ into $K$.

Recall that an analytic reproducing kernel Hilbert space on the disk is a Hilbert space $H$ of analytic functions on the disk such that for each $|w|<1$ the functional $f \mapsto f(w)$ is bounded on $H$. By the Riesz Representation Theorem there exist functions $k_{w} \in H$ such that $f(w)=\left\langle f, k_{w}\right\rangle_{H}$ for all $w$ in the disk. The function $K(z, w)=k_{w}(z)$ is called the kernel function for $H$ and we will write $H(K)$ instead of $H$ since the kernel uniquely determines the Hilbert space $H$.

Corollary 1. Let $H(K)$ be an analytic reproducing kernel Hilbert space on the disk such that $T f(z) \equiv z f(z)$ is polynomially bounded on $H(K)$. Let $\overline{H(K)}$ denote the Hilbert module $\left(H(K), T^{*}\right)$. Then $\operatorname{Ext}_{A(D)}\left(\overline{H(K)}, H^{2}\right)$ can be identified with $\mathcal{M}\left(H^{2}, H(K)\right)$, the set of pointwise multipliers from $H^{2}$ into $H(K)$.

Proof. Suppose $1 \otimes g=S Y-Y T^{*}$ for some bounded $Y$. Multiplying on the left by $S^{*}$ yields $Y=S^{*} Y T^{*}$. So $\forall|w|<1,0=\left(Y-S^{*} Y T^{*}\right) k_{w}=\left(1-\bar{w} S^{*}\right) Y k_{w}$. Therefore, $Y k_{w}=0 \quad \forall|w|<1$. Since the kernel functions $k_{w}$ span $H(K), Y=0$. It follows from remark (c) above that $\operatorname{Ext}_{A(D)}\left(\overline{H(K)}, H^{2}\right)$ can be identified with the space of functions $g \in H^{2}(\beta)$ such that $p \mapsto p(T) g$ extends to a bounded operator 
from $H^{2}$ into $H^{2}(\beta)$. It is easy to show, using the Closed Graph Theorem together with the fact that the evaluation functionals are continuous on both $H^{2}$ and $H(K)$, that $g \in \mathcal{M}\left(H^{2}, H^{2}(\beta)\right)$.

Let $\left\{\beta_{n}\right\}$ be a sequence of positive numbers with $\beta_{0}=1$ and such that $\sup _{n \geq 0} \beta_{n} / \beta_{n+1}<\infty$. Then $H^{2}(\beta)$ is defined to be the Hilbert space of analytic functions $f(z)=\sum_{n=0}^{\infty} a_{n} z^{n}$ such that

$$
\|f\|^{2} \equiv \sum_{n=0}^{\infty} \frac{\left|a_{n}\right|^{2}}{\beta_{n}}<\infty
$$

It is well known, [7], that $T f(z)=z f(z)$ is bounded on $H^{2}(\beta)$ and unitarily equivalent to the weighted shift on $H^{2}$ with weight sequence $\left\{\sqrt{\beta_{n} / \beta_{n+1}}\right\}$. Throughout, we will assume that $T$ is a contraction so that $\left(H^{2}(\beta), T\right)$ is a Hilbert A(D)-module.

We will use the following notation in the proposition below. For $g \in H^{2}, \Gamma_{g}$ will denote the Hankel matrix with symbol $g$. That is

$$
\Gamma_{g}=(\hat{g}(i+j))_{i, j \geq 0},
$$

where $\hat{g}(n)$ are the Fourier coefficients of $g$.

For $\varphi \in L^{\infty}(T)$, let $T_{\varphi}$ denote the Toeplitz matrix

$$
T_{\varphi}=(\hat{\varphi}(i-j))_{i, j \geq 0} .
$$

Finally, $D_{\beta}$ will denote the diagonal matrix, $\operatorname{diag}\left(\sqrt{\beta_{n}}\right)_{n \geq 0}$.

Proposition 1. For $g$ in $H^{2}(\beta)$, define $g_{1}$ in $H^{2}$ by $\hat{g_{1}}(n)=\hat{g}(n) / \beta_{n}$. Let

$$
R(1 \otimes g)=\left(\begin{array}{cc}
S & 1 \otimes g \\
0 & T
\end{array}\right) .
$$

(1) $R(1 \otimes g)$ is polynomially bounded on $H^{2} \oplus H^{2}(\beta)$ if and only if the weighted Hankel matrix $D_{\beta} \Gamma_{g_{1}}$ is bounded on $\ell^{2}$.

(2) There is an operator $Y$ in $\mathcal{L}\left(H^{2}(\beta), H^{2}\right)$ such that $1 \otimes g=S Y-Y T$ if and only if $\exists \varphi \in L^{\infty}(\mathbf{T})$ satisfying

a) $P_{+}\left(\overline{e^{i \theta} \varphi\left(e^{i \theta}\right)}\right)=-g_{1}$, and

b) The weighted Toeplitz matrix $T_{\varphi} D_{\beta}$ is bounded on $\ell^{2}$.

Proof. By remark (c), the matrix $R(1 \otimes g)$ is polynomially bounded if and only if the operator $W e_{j}=T^{* j} g$ extends to a bounded operator from $H^{2}$ into $H^{2}(\beta)$. One checks that the matrix for this operator with respect to the usual orthonormal bases, $\left\{e_{n}\right\}_{n \geq 0}$ for $H^{2}$ and $\left\{\sqrt{\beta_{n}} z^{n}\right\}_{n \geq 0}$ for $H^{2}(\beta)$, is the weighted Hankel matrix above. For the proof of (2), suppose $1 \otimes g=S Y-Y T$ for some $Y \in \mathcal{L}\left(H^{2}(\beta), H^{2}\right)$. Multiplying on the left by $S^{*}$, we see that $Y=S^{*} Y T$. It follows that the matrix $\left(\left\langle Y z^{j}, e_{i}\right\rangle_{2}\right)_{i, j \geq 0}$ is Toeplitz. To see that the matrix is bounded, note that $T$ is a contraction so that $D_{\beta}{ }^{-1}$ is bounded. Hence $D_{\beta}{ }^{-1} Y$ is bounded. But this is the Toeplitz matrix above. From the commutator equation one has $\hat{g}_{1}(n)=$ $-\left\langle Y^{*} e_{0}, z^{n+1}\right\rangle_{\beta}$.

Corollary 2. $\operatorname{Ext}_{A(D)}\left(H^{2}, H^{2}\right)=(0)$.

Proof. By setting $\beta_{n}=1$, one sees that $R(1 \otimes g)$ is polynomially bounded if and only if the Hankel matrix $\Gamma_{g}$ is bounded on $\ell^{2}$. By Nehari's theorem, this happens if and only if $g=P_{+} \psi$ for some $\psi \in L^{\infty}(\mathbf{T})$. 
Corollary 3. If $\beta_{n} \longrightarrow+\infty$, then we may identify $\operatorname{Ext}_{A(D)}\left(H^{2}(\beta), H^{2}\right)$ as the vector space of functions $g \in H^{2}(\beta)$ such that the matrix $D_{\beta} \Gamma_{g_{1}}$ is bounded on $\ell^{2}$.

Proof. Note that the diagonal matrix $D_{\beta}^{-1}=\operatorname{diag}\left(\beta_{j}^{-1 / 2}\right)_{j \geq 0}$ is compact. So if $T_{\varphi} D_{\beta}$ is bounded, then $T_{\varphi}$ is compact and this implies $\varphi=0$. Therefore, no nonzero rank one operator of the form $1 \otimes g$ is a commutator.

Remark. If we take $\beta_{n}=n+1$, then $H^{2}(\beta)$ is the Bergman space, $L_{a}^{2}$, and $g^{\prime} \in$ $\operatorname{Ext}_{A(D)}\left(L_{a}^{2}, H^{2}\right)$ if and only if the matrix $\operatorname{diag}(\sqrt{n+1})_{n>0} \Gamma_{g}$ is bounded on $\ell^{2}$. This happens if and only if the range of $\Gamma_{g}$ is contained in the Dirichlet space $=H^{2}\left(\frac{1}{\beta}\right)$. It is not hard to see that $\operatorname{Ext}_{A(D)}\left(L_{a}^{2}, H^{2}\right)$ contains all Bloch functions. In fact, if $T$ is pointwise multiplication by $z$ on $L_{a}^{2}$, then the operator $W$ defined on polynomials by $W(p)=p\left(T^{*}\right) g$ is just the restriction of the little Hankel (with symbol $g$ ) on the Bergman space to $H^{2}$. It is well known that this operator is bounded on $L_{a}^{2}$ if and only if $g$ is Bloch, see [8]. It will follow from a result in the next section that $\mathcal{M}\left(H^{2}, L_{a}^{2}\right)$ is also contained in $\operatorname{Ext}_{A(D)}\left(L_{a}^{2}, H^{2}\right)$.

\section{A PROOF OF Bourgain's RESUlt}

Bourgain in [1] proved that the operator

$$
R_{f}=\left(\begin{array}{cc}
S^{*} & \Gamma_{f} \\
0 & S
\end{array}\right)
$$

is similar to a contraction if $f^{\prime} \in$ BMOA. In this section we will give an alternative proof of this result using subnormality and a characterization of BMOA in terms of Carleson measures on the Hardy space. What we will prove is the following:

$$
f^{\prime} \text { is BMOA } \Longrightarrow \text { the matrix } A=((i+1) \hat{f}(i+j))_{i, j \geq 0} \text { is bounded on } \ell^{2} \text {. }
$$

Note that if $A$ is bounded, then $\Gamma_{f}=A-S A S=S^{*}(S A)-(S A) S$. Hence, $R_{f}$ is similar to $S^{*} \oplus S$ via the similarity

$$
\left(\begin{array}{cc}
I & S A \\
0 & I
\end{array}\right)
$$

Proof of (2).

Lemma 1. Suppose that $T$ is subnormal on a Hilbert space $K$ and let $f \in K$. If $W(p) \equiv p(T) f$ extends to a bounded operator from $H^{2}$ into $K$, then the operator $V(p) \equiv p\left(T^{*}\right) f$ extends to a bounded operator from $H^{2}$ into $K$.

Proof. The transpose of the matrix for $W^{*} W$ is

$$
B=\left(\left\langle T^{* j} T^{i} f, f\right\rangle_{K}\right)_{i, j \geq 0}
$$

and the the matrix for $V^{*} V$ is

$$
C=\left(\left\langle T^{i} T^{* j} f, f\right\rangle_{K}\right)_{i, j \geq 0} .
$$

By the Bram-Halmos criterion for subnormality, $B \geq C$. 
Remark. If $T f(z) \equiv z f(z)$ is polynomially bounded on $H^{2}(\beta)$ and $\beta_{n} \longrightarrow \infty$, then by Corollary 3.2, $\operatorname{Ext}_{A(D)}\left(H^{2}(\beta), H^{2}\right)$ is the vector space of functions $g \in H^{2}(\beta)$ such that $p \mapsto p\left(T^{*}\right) g$ extends to a bounded operator from $H^{2}$ into $H^{2}(\beta)$. If we suppose further that $T$ is subnormal, then it follows from Lemma 4.1 that $\mathcal{M}\left(H^{2}, H^{2}(\beta)\right)$ is contained in $\operatorname{Ext}_{A(D)}\left(H^{2}(\beta), H^{2}\right)$.

By Nehari's theorem a function $f \in H^{2}$ is BMOA if and only if the Hankel matrix $\Gamma_{f}$ is bounded on $\ell^{2}$. Another useful criterion for $f$ to be BMOA is that the measure $\left|f^{\prime}(z)\right|^{2} \log \frac{1}{|z|} d A(z)$ is Carleson for $H^{2}$, where $A$ is normalized area measure on the disk. A good reference for this is Zhu's book [8]. Now what this means is that $f^{\prime}$ is a pointwise multiplier from $H^{2}$ into $\mathcal{P}^{2}(\mu)$, the closure of the analytic polynomials in $L^{2}(\mu)$, where $d \mu(z)=\log \frac{1}{|z|} d A(z)$. One verifies that $\frac{1}{(n+1)^{2}}=\int_{D}|z|^{2 n} \log \frac{1}{|z|} d A(z)$ so that $\mathcal{P}^{2}(\mu)=H^{2}(\beta)$ with $\beta_{n}=(n+1)^{2}$, and multiplication by $z$ is subnormal on this space. By the remark above $f^{\prime} \in \operatorname{Ext}_{A(D)}\left(H^{2}(\beta), H^{2}\right)$. So by Proposition 4.1, the matrix

$$
\left(\frac{i+1}{i+j+1} \hat{f}(i+j)\right)_{i, j \geq 0}
$$

must be bounded on $\ell^{2}$. If we repeat this argument with $f^{\prime}$ in place of $f$, we get that the matrix $A$ is bounded.

\section{ACKNOWLEDGEMENTS}

I would like to thank my advisor, Vern Paulsen, for his guidance and advice while working on this project.

\section{REFERENCES}

[1] J. Bourgain, The Similarity Problem for polynomially bounded operators on Hilbert space, Israel Journal of Math., Vol. 54, No. 2, (1986). MR 88h:47024

[2] J. F. Carlson and D. N. Clark, Cohomology and extensions of Hilbert modules, J.Functional Analysis 128 (1995).

[3] J. F. Carlson, D. N. Clark, C. Foias and J. P. Williams, Projective Hilbert A(D)-Modules, New York J. Math. 1 (1994), 26-38. CMP 94:15

[4] R. G. Douglas and V. I. Paulsen, Hilbert modules over function algebras, Longman Scientific \& Technical, New York (1989). MR 91g:46084

[5] C. Foias and J. P. Williams, On a class of polynomially bounded operators, preprint (1974).

[6] V. V. Peller, Estimates of functions of power bounded operators on Hilbert spaces, J. Operator Theory, 7 (1982), 341-372. MR 83i:47019

[7] A. Shields, Weighted shift operators and analytic function theory, Topics in Operator Theory, Math. Surveys, No. 13, Amer. Math. Soc., Providence, R.I. (1974) MR 50:14341

[8] K. Zhu, Operator Theory in function spaces, Marcel Dekker, Inc., New York and Basel (1990). MR 92c: 47031

Department of Mathematics, University of Houston, Houston, Texas 77204-3476

E-mail address: sarah@math.uh.edu 